\title{
Distributions of the Maximum Likelihood and Minimum Contrast Estimators Associated with the Fractional Ornstein-Uhlenbeck Process
}

\author{
Katsuto Tanaka \\ Department of Economics, Hitotsubashi University \\ tanaka@stat.hit-u.ac.jp
}

August, 2011

\begin{abstract}
We discuss some inference problems associated with the fractional Ornstein-Uhlenbeck (fO-U) process driven by the fractional Brownian motion (fBm). In particular, we are concerned with the estimation of the drift parameter, assuming that the Hurst parameter $H$ is known and is in $[1 / 2,1)$. Under this setting we compute the distributions of the maximum likelihood estimator (MLE) and the minimum contrast estimator (MCE) for the drift parameter, and explore their distributional properties by paying attention to the influence of $H$ and the sampling span $M$. We shall also derive the asymptotic distributions of the two estimators as $M$ becomes large. We further deal with the ordinary least squares estimator (OLSE) and examine the asymptotic relative efficiency. It is shown that the MCE is asymptotically efficient, while the OLSE is inefficient. We also consider the unit root testing problem in the fO- $U$ process and compute the power of the tests based on the MLE and MCE.
\end{abstract}




\section{Introduction}

The present paper is concerned with the inference problem associated with the fractional Ornstein-Uhlenbeck (fO-U) process $\left\{Y_{H}(t)\right\}$ defined by

$$
Y_{H}(t)=\alpha \int_{0}^{t} Y_{H}(s) d s+B_{H}(t), \quad(0 \leq t \leq M),
$$

where $\alpha(\alpha \leq 0)$ is the drift parameter, whereas $\left\{B_{H}(t)\right\}$ is the fractional Brownian motion $(\mathrm{fBm})$ with the Hurst parameter $H$ defined shortly. It is assumed that $\left\{Y_{H}(t)\right\}$ is continuously observed in the time interval $[0, M]$.

The fO-U process in (1) is often expressed as the following differential form:

$$
d Y_{H}(t)=\alpha Y_{H}(t) d t+d B_{H}(t), \quad Y_{H}(0)=0, \quad(0 \leq t \leq M) .
$$

We can solve (1) for $Y_{H}(t)$ to get

$$
Y_{H}(t)=e^{\alpha t} \int_{0}^{t} e^{-\alpha s} d B_{H}(s)
$$

where the stochastic integral exists as a path-wise Riemann-Stieltjes integral and the solution is unique (Cheridito, Kawaguchi and Maejima 2003).

The fBm $\left\{B_{H}(t)\right\}$ was invented by Kolmogorov (1940) and was largely developed by Mandelbrot and Van Ness (1968), who obtained the following integral representation:

$$
B_{H}(t)=c_{H}\left[\int_{-\infty}^{0}\left\{(t-u)^{H-1 / 2}-(-u)^{H-1 / 2}\right\} d W(u)+\int_{0}^{t}(t-u)^{H-1 / 2} d W(u)\right],
$$

where $c_{H}=(2 H \Gamma(3 / 2-H) /(\Gamma(H+1 / 2) \Gamma(2-2 H)))^{1 / 2}$ with $\Gamma(z)$ being the gamma function, whereas $\{W(t)\}$ is the standard Bm. We assume that the Hurst parameter $H$ is in $[1 / 2,1)$ and is assumed to be known throughout this paper. Note that, when $H=1 / 2$, the $\mathrm{fBm}$ reduces to the standard $\mathrm{Bm}$, that is, $B_{1 / 2}(t)=W(t)$, and the fO- $\mathrm{U}$ process in (1) reduces to the ordinary $\mathrm{O}-\mathrm{U}$ process.

The covarince kernel of $\left\{B_{H}(t)\right\}$ is given by

$$
K_{H}(s, t)=\operatorname{Cov}\left(B_{H}(s), B_{H}(t)\right)=\frac{1}{2}\left(s^{2 H}+t^{2 H}-|s-t|^{2 H}\right),
$$

which implies that the $\mathrm{fBm}$ is self-similar with the self-similarity parameter $H$, that is, $\left\{B_{H}(\gamma t)\right\} \stackrel{\mathcal{D}}{=}\left\{\gamma^{H} B_{H}(t)\right\}$ for any $\gamma>0$, where $\stackrel{\mathcal{D}}{=}$ denotes the distributional equivalence. The fO- $U$ process is also self-similar because of (3).

It follows from (5) that the fBm has stationary increments in the sense that

$$
\mathrm{V}\left(B_{H}(t)-B_{H}(s)\right)=|s-t|^{2 H}, \quad(1 / 2 \leq H<1),
$$

but, when $1 / 2<H<1$, the increments are not independent and are positively correlated, unlike the standard Bm. In fact, puttting $\Delta B_{H}(k)=B_{H}(k)-B_{H}(k-1)$ for each $k=1,2, \cdots$, it holds that

$$
\begin{aligned}
r(n) & =\operatorname{Cov}\left(\Delta B_{H}(k), \Delta B_{H}(k+n)\right)=\frac{1}{2}\left[(n+1)^{2 H}+(n-1)^{2 H}-2 n^{2 H}\right] \\
& \sim H(2 H-1) n^{2 H-2}, \quad(n=1,2, \cdots),
\end{aligned}
$$


which further implies that the increments of the fBm have the long-range dependence property in the sense that

$$
\sum_{n=1}^{\infty} r(n)=\sum_{n=1}^{\infty} \operatorname{Cov}\left(\Delta B_{H}(k), \Delta B_{H}(k+n)\right)=\infty, \quad(1 / 2<H<1) .
$$

It is known that the $\mathrm{fBm}$ and $\mathrm{fO}-\mathrm{U}$ processes are neither a Markov process nor a semimartingale. As was shown in Gripenberg and Norros (1996), the following infinitesimal rule holds:

$$
\operatorname{Cov}\left(d B_{H}(s), d B_{H}(t)\right)=H(2 H-1)|s-t|^{2 H-2} d s d t, \quad(1 / 2<H<1) .
$$

This contrasts with the stadard Bm in which the increments are independent. This fact is closely related with the nonsemimartingale nature of the $\mathrm{fBm}$ and $\mathrm{fO}-\mathrm{U}$, which makes various computations complicated. As an example, it is difficult to compute explicitly

$$
\mathrm{V}\left(Y_{H}(t)\right)=H(2 H-1) e^{2 \alpha t} \int_{0}^{t} \int_{0}^{t} e^{-\alpha(r+s)}|r-s|^{2 H-2} d r d s, \quad(1 / 2<H<1),
$$

whereas, when $H=1 / 2$, we have

$$
\mathrm{V}\left(Y_{1 / 2}(t)\right)=e^{2 \alpha t} \int_{0}^{t} \int_{0}^{t} e^{-\alpha(r+s)} \mathrm{E}(d W(r) d W(s))=e^{2 \alpha t} \int_{0}^{t} e^{-2 \alpha s} d s=\frac{e^{2 \alpha t}-1}{2 \alpha} .
$$

The fO-U process arises naturally from the discrete-time near unit root process whose innovation error follows a long-memory process. More specifically, let us consider

$$
y_{j}=\rho y_{j-1}+v_{j},(1-L)^{H-1 / 2} v_{j}=\varepsilon_{j}, \rho=1+\frac{\alpha}{T}, y_{0}=0,(j=1, \cdots, T) .
$$

where $L$ is the lag-operator, $\left\{\varepsilon_{j}\right\} \sim$ i.i.d. $(0,1)$, whereas $\left\{v_{j}\right\}$ is a stationary long-memory process generated by

$$
v_{j}=(1-L)^{-(H-1 / 2)} \varepsilon_{j}=\sum_{k=0}^{\infty} \frac{\Gamma(k+H-1 / 2)}{\Gamma(H-1 / 2) \Gamma(k+1)} \varepsilon_{j-k}, \quad(1 / 2<H<1) .
$$

Then it can be shown that

$$
\frac{c_{H} \Gamma(H+1 / 2)}{T^{H}} y_{[T t]} \Rightarrow Y_{H}(t), \quad(0 \leq t \leq 1),
$$

where $\Rightarrow$ signifies weak convergence as $T \rightarrow \infty$.

The main purpose of the present paper is to discuss the estimation problem of the drift parameter $\alpha$ in the fO- $\mathrm{U}$ process in (1) or (2), assuming the Hurst parameter $H$ $(1 / 2 \leq H<1)$ to be known. This problem was earlier discussed in Kleptsyna and Le Breton (2002) and $\mathrm{Hu}$ and Nualart (2010). The former derives the maximum likelihood estimator (MLE) on the basis of the fractional version of Girsanov's theorem, whereas the latter establishes some asymptotic properties of the ordinary least squares estimator (OLSE). In this paper we are more concerned with computational aspects and compute the distribution of the MLE. We also take up the minimum contrast estimator (MCE) dealt with in Bishwal (2008) and compute its distribution as well. The asymptotic distributions of the MLE and MCE as the sampling span $M \rightarrow \infty$ are derived. We also consider the testing problem $H_{0}: \alpha=0$ against $H_{1}: \alpha<0$, which is interpreted as the unit root test for the discrete-time model in (8). 
This paper is organized as follows. Section 2 defines the OLSE, MLE and MCE of $\alpha$ and gives a brief discussion of each estimator. We also give the characteristic functions (c.f.s) associated with the MLE and MCE, but the derivation of the c.f. associated with the OLSE is much involved and remains to be done. Section 3 computes numerically the distributions of the MLE and MCE together with their moments, and presents graphs of those densities for various values of $\alpha, H$ and $M$. We also derive the asymptotic distributions of the two estimators. Section 4 considers testing $H_{0}: \alpha=0$ against $H_{1}: \alpha<0$, and computes the powers of the tests based on the MLE and MCE. Section 5 concludes this paper. Proofs of theorems are given in the Appendix.

\section{OLSE, MLE and MCE of $\alpha$}

In this section we discuss the OLSE, MLE and MCE of $\alpha$ for the fO-U process in (1) or (2). We first deal with the OLSE in Section 2.1, where only the asymptotic properties as $M \rightarrow \infty$ are discussed together with the difference from the OLSE of the coefficient $\rho$ in the discrete-time model (8), whereas the MLE and MCE are discussed in Section 2.2, where the c.f.s are given to compute numerically their distributions in the next section.

\subsection{Asymptotic properties of the OLSE}

The OLS estimation of $\alpha$ was discussed in $\mathrm{Hu}$ and Nualart (2010). The OLSE $\hat{\alpha}_{1}$ can be obtained formally from (2) as

$$
\hat{\alpha}_{1}=\frac{\int_{0}^{M} Y_{H}(t) d Y_{H}(t)}{\int_{0}^{M} Y_{H}^{2}(t) d t}=\alpha+\frac{\int_{0}^{M} Y_{H}(t) d B_{H}(t)}{\int_{0}^{M} Y_{H}^{2}(t) d t} .
$$

Here the stochastic integral with respect to $B_{H}(t)$ is not the ordinary Ito integral because $B_{H}(t)$ is not a semimartingale. This integral is defined as follows. Let us denote by $p_{m}$ an arbitray partition of the interval $[0, M]$, where $p_{m}: 0=t_{0}<t_{1}<\cdots<t_{m}=M$. Then consider the following sum:

$$
S\left(Y_{H}, p_{m}\right)=\sum_{i=0}^{m-1} Y_{H}\left(t_{i}\right) \diamond\left(B_{H}\left(t_{i+1}\right)-B_{H}\left(t_{i}\right)\right),
$$

where ' $\diamond$ ' is called the Wick product (see Duncan, Hu and Pasik-Duncan (2000) for its definition and various properties). Then we define, putting $\Delta_{m}=\max \left(t_{i}-t_{i-1}\right)$,

$$
\int_{0}^{M} Y_{H}(t) d B_{H}(t)=\lim _{\Delta_{m} \rightarrow 0} \sum_{i=0}^{m-1} Y_{H}\left(t_{i}\right) \diamond\left(B_{H}\left(t_{i+1}\right)-B_{H}\left(t_{i}\right)\right)
$$

Because the Wick product has the property that $\mathrm{E}\left(Y_{H}\left(t_{i}\right) \diamond\left(B_{H}\left(t_{i+1}\right)-B_{H}\left(t_{i}\right)\right)\right)=$ $\mathrm{E}\left(Y_{H}\left(t_{i}\right)\right) \mathrm{E}\left(B_{H}\left(t_{i+1}\right)-B_{H}\left(t_{i}\right)\right)=0$, it holds that

$$
\mathrm{E}\left(\int_{0}^{M} Y_{H}(t) d B_{H}(t)\right)=0 .
$$

In particular, it holds that

$$
\int_{0}^{M} B_{H}(t) d B_{H}(t)=\frac{1}{2}\left(B_{H}^{2}(M)-M\right), \quad(1 / 2<H<1),
$$

which also holds when $H=1 / 2$. 
On the other hand there is another definition of the stochastic integral, which is of Stratonovich type. We denote it as

$$
\int_{0}^{M} Y_{H}(t) \delta B_{H}(t)=\lim _{\Delta_{m} \rightarrow 0} \sum_{i=0}^{m-1} Y_{H}\left(t_{i}\right)\left(B_{H}\left(t_{i+1}\right)-B_{H}\left(t_{i}\right)\right) .
$$

In particular, it holds that

$$
\int_{0}^{M} B_{H}(t) \delta B_{H}(t)=\frac{1}{2} B_{H}^{2}(M), \quad(1 / 2<H<1) .
$$

In general, the integrals of Ito type and Stratonovich type are different and the following relationship holds:

$$
\int_{0}^{M} Y_{H}(t) \delta B_{H}(t)=\int_{0}^{M} Y_{H}(t) d B_{H}(t)+\mathrm{E}\left(\int_{0}^{M} Y_{H}(t) \delta B_{H}(t)\right) .
$$

More specifically, it was shown in $\mathrm{Hu}$ and Nualart (2010) that

$$
\begin{aligned}
& A_{H}(M)=\mathrm{E}\left(\int_{0}^{M} Y_{H}(t) \delta B_{H}(t)\right)=H(2 H-1) \int_{0}^{M} \int_{0}^{t} u^{2 H-2} e^{\alpha u} d u d t \\
& \int_{0}^{M} Y_{H}(t) \delta B_{H}(t)=-\alpha \int_{0}^{M} Y_{H}^{2}(t) d t+\frac{1}{2} Y_{H}^{2}(M), \\
& \int_{0}^{M} Y_{H}(t) d B_{H}(t)=-\alpha \int_{0}^{M} Y_{H}^{2}(t) d t+\frac{1}{2} Y_{H}^{2}(M)-A_{H}(M) .
\end{aligned}
$$

It follows from the above arguments that

$$
\hat{\alpha}_{1}=\frac{Y_{H}^{2}(M) / 2-A_{H}(M)}{\int_{0}^{M} Y_{H}^{2}(t) d t},
$$

where $\mathrm{Hu}$ and Nualart (2010) proved that, when $\alpha<0$,

$$
\begin{aligned}
& \frac{1}{M} Y_{H}^{2}(M) \rightarrow 0, \\
& \lim _{M \rightarrow \infty} A_{H}(M)=H(2 H-1)(-\alpha)^{1-2 H} \Gamma(2 H-1), \\
& \frac{1}{M} \int_{0}^{M} Y_{H}^{2}(t) d t \rightarrow \alpha^{-2 H} H \Gamma(2 H),
\end{aligned}
$$

where the convergence in (16) and (18) holds almost surely and in mean square. It follows that, as $M \rightarrow \infty$,

$$
\hat{\alpha}_{1} \rightarrow \frac{-H(2 H-1)(-\alpha)^{1-2 H} \Gamma(2 H-1)}{\alpha^{-2 H} H \Gamma(2 H)}=\alpha, \quad(\alpha<0),
$$

almost surely and in mean square.

$\mathrm{Hu}$ and Nualart (2010) further proved the asymptotic normality of $\hat{\alpha}_{1}$. It was shown that, when $1 / 2 \leq H<3 / 4$ and $\alpha<0$, it holds that

$$
\sqrt{M}\left(\hat{\alpha}_{1}-\alpha\right) \rightarrow \mathrm{N}\left(0,-\alpha \sigma_{H}^{2}\right), \quad \sigma_{H}^{2}=(4 H-1)\left(1+\frac{\Gamma(3-4 H) \Gamma(4 H-1)}{\Gamma(2-2 H) \Gamma(2 H)}\right) .
$$


It can be checked that $\sigma_{H}^{2}$ increases monotonically from 2 as $H$ gets away from $H=1 / 2$. The asymptotic efficiency of $\hat{\alpha}_{1}$ will be compared with the MLE later.

It is of some interest to consider another OLSE $\hat{\alpha}_{2}$ based on the integral of Stratnovich type, which is

$$
\hat{\alpha}_{2}=\frac{Y_{H}^{2}(M) / 2}{\int_{0}^{M} Y_{H}^{2}(t) d t}=\alpha+\frac{\int_{0}^{M} Y_{H}(t) \delta B_{H}(t)}{\int_{0}^{M} Y_{H}^{2}(t) d t} .
$$

Note that this estimator is closely related with the OLSE $\hat{\rho}$ of $\rho$ in the discrete-time near unit root process (8). In fact, when $1 / 2<H<1$, it holds that, as $T \rightarrow \infty$,

$$
\begin{aligned}
T(\hat{\rho}-1) & =\frac{1}{T^{2 H}} \sum_{j=2}^{T} y_{j-1}\left(y_{j}-y_{j-1}\right) / \frac{1}{T^{2 H+1}} \sum_{j=2}^{T} y_{j-1}^{2} \\
& \Rightarrow \frac{Y_{H}^{2}(1) / 2}{\int_{0}^{1} Y_{H}^{2}(t) d t} \stackrel{\mathcal{D}}{=} M \frac{Y_{H}^{2}(M) / 2}{\int_{0}^{M} Y_{H}^{2}(t) d t} .
\end{aligned}
$$

This last distributional equivalence comes from the self-similarity property of $\left\{Y_{H}(t)\right\}$. Note that, because of $\rho=1+(\alpha / T)$ so that $\alpha=T(\rho-1)$, the limiting distibution of $T(\hat{\rho}-1)$ may be interpreted as that of the OLSE of $\alpha$ in $\rho=1+(\alpha / T)$.

Returning to the OLSE in (20), it turns out that, when $\alpha<0, \hat{\alpha}_{2} \rightarrow 0$ almost surely and in mean square as $M \rightarrow \infty$ because of (16) and (18). Thus $\hat{\alpha}_{2}$ is not consistent for $\alpha<0$. When $\alpha=0, \hat{\alpha}_{2}$ is consistent because

$$
\hat{\alpha}_{2} \stackrel{\mathcal{D}}{=} \frac{1}{M} \frac{Y_{H}^{2}(1) / 2}{\int_{0}^{1} Y_{H}^{2}(t) d t} .
$$

On the basis of the fact described in (18), Hu and Nualart (2010) suggested a practical estimator defined by

$$
\hat{\alpha}_{3}=-\left(\frac{1}{H \Gamma(2 H) M} \int_{0}^{M} Y_{H}^{2}(t) d t\right)^{-1 / 2 H},
$$

and proved that, when $1 / 2<H<3 / 4$,

$$
\sqrt{M}\left(\hat{\alpha}_{3}-\alpha\right) \rightarrow \mathrm{N}\left(0,-\frac{\alpha}{4 H^{2}} \sigma_{H}^{2}\right)
$$

where $\sigma_{H}^{2}$ is defined in (19). It is seen that the estimator $\hat{\alpha}_{3}$ attains lower variances asymptotically than $\hat{\alpha}_{1}$. When $H=1 / 2$, this estimator is known as the MCE, which we discuss in the next subsection.

\subsection{Derivation of the c.f.s associated with the MLE and MCE}

To consider the MLE $\hat{\alpha}$ we follow Kleptsyna and Le Breton (2002). Let us rewrite $Y_{H}(t)$ in $(2)$ as $Y_{H}^{\alpha}(t)$ so that the dependence on $\alpha$ is explicit. Then the differential equation (2) becomes

$$
d Y_{H}^{\alpha}(t)=\alpha Y_{H}^{\alpha}(t) d t+d B_{H}(t), \quad Y_{H}^{\alpha}(0)=0, \quad(0 \leq t \leq M) .
$$

We also define the auxiliary process

$$
d Y_{H}^{\beta}(t)=\beta Y_{H}^{\beta}(t) d t+d B_{H}(t), \quad Y_{H}^{\beta}(0)=0, \quad(0 \leq t \leq M),
$$


where $\beta$ is a parameter to be determined later.

Let $(C[0, M], \mathcal{B}(C))$ be the measurable space of continuous functions on $[0, M]$ with the associated $\sigma$-field generated under teh supremum norm. Let $\mu_{Y_{H}^{\alpha}}$ and $\mu_{Y_{H}^{\beta}}$ be the probability measures on $(C[0, M], \mathcal{B}(C))$ induced by $\left\{Y_{H}^{\alpha}(t)\right\}$ and $\left\{Y_{H}^{\beta}(t)\right\}$, respectively, by the relation

$$
\mu_{Y_{H}^{\alpha}}(A)=P\left(\omega: Y_{H}^{\alpha} \in A\right), \quad \mu_{Y_{H}^{\beta}}(A)=P\left(\omega: Y_{H}^{\beta} \in A\right), \quad A \in \mathcal{B}(C) .
$$

Then the fractional version of Girsanov's theorem was obtained by Kleptsyna, Le Breton and Roubaud (2000), which says that measures $\mu_{Y_{H}^{\alpha}}$ and $\mu_{Y_{H}^{\beta}}$ are equivalent and the Radon-Nikodym derivative evaluated at $Y_{H}^{\gamma}=\left\{Y_{H}^{\gamma}(t)\right\} \in C[0, M]$ is given by

$$
\frac{d \mu_{Y_{H}^{\alpha}}}{d \mu_{Y_{H}^{\beta}}}\left(Y_{H}^{\gamma}\right)=\exp \left[(\alpha-\beta) \int_{0}^{M} Q_{H}^{\gamma}(t) d Z_{H}^{\gamma}(t)-\frac{\alpha^{2}-\beta^{2}}{2} \int_{0}^{M}\left\{Q_{H}^{\gamma}(t)\right\}^{2} d v_{H}(t)\right] \text {, }
$$

where $v_{H}(t)=t^{2-2 H} \Gamma(3 / 2-H) /(2 H \Gamma(3-2 H) \Gamma(H+1 / 2))$, and

$$
\begin{aligned}
Q_{H}^{\gamma}(t) & =\frac{d}{d v_{H}(t)} \int_{0}^{t} g_{H}(t, s) Y_{H}^{\gamma}(s) d s \\
& =\frac{\eta_{H}}{4(1-H)}\left\{t^{2 H-1} Z_{H}^{\gamma}(t)+\int_{0}^{t} s^{2 H-1} d Z_{H}^{\gamma}(s)\right\}, \\
Z_{H}^{\gamma}(t) & =\int_{0}^{t} g_{H}(t, s) d Y_{H}^{\gamma}(s),
\end{aligned}
$$

with $\eta_{H}=2 H \Gamma(3-2 H) \Gamma(H+1 / 2) / \Gamma(3 / 2-H)$ and $g_{H}(t, s)=(2 H \Gamma(3 / 2-H) \Gamma(H+$ $1 / 2))^{-1}(s(t-s))^{1 / 2-H}$.

It is shown in Kleptsyna and Le Breton (2002) that the sample paths of the process $\left\{Q_{H}^{\gamma}(t)\right\}$ in $(27)$ belong to $L^{2}\left([0, M], d v^{H}\right)$, and the process $\left\{Z_{H}^{\gamma}(t)\right\}$ in $(28)$ is a Gaussian semimartingale with the decomposition

$$
Z_{H}^{\gamma}(t)=\gamma \int_{0}^{t} Q_{H}^{\gamma}(t) d v_{H}(t)+M_{H}(t), \quad M_{H}(t)=\int_{0}^{t} g_{H}(t, s) d B_{H}(s),
$$

where $\left\{M_{H}(t)\right\}$ is shown to be a Gaussian martingale by Norros, Valkeila and Virtamo (1999).

The Radon-Nikodym derivative in (26) is composed of various complicated processes and contain two types of integrals. One is the Ito integral with respect to a semimartingale and the other the ordinary Rieman-Stieltjes integral. Suppose that $H=1 / 2$. Then it is seen that we have $v_{1 / 2}(t)=t$ and $Q_{1 / 2}^{\gamma}(t)=Z_{1 / 2}^{\gamma}(t)=Y_{1 / 2}^{\gamma}(t)$ so that both $Q_{1 / 2}^{\gamma}(t)$ and $Z_{1 / 2}^{\gamma}(t)$ follow the same ordinary $\mathrm{O}-\mathrm{U}$ process as $Y_{1 / 2}^{\gamma}(t)$. Thus the Radon-Nikodym derivative reduces to

$$
\frac{d \mu_{Y_{1 / 2}^{\alpha}}}{d \mu_{Y_{1 / 2}^{\beta}}}\left(Y_{1 / 2}^{\gamma}\right)=\exp \left[(\beta-\alpha) \int_{0}^{1} Y_{1 / 2}^{\gamma}(t) d Y_{1 / 2}^{\gamma}(t)-\frac{\alpha^{2}-\beta^{2}}{2} \int_{0}^{1}\left\{Y_{1 / 2}^{\gamma}(t)\right\}^{2} d t\right] .
$$

This formula was initially given by Liptser and Shiryaev (1977).

Now we can define the likelihood for $\alpha$ as $l(\alpha)=d \mu_{Y_{H}^{\alpha}}\left(Y_{H}^{\alpha}\right) / d \mu_{Y_{H}^{0}}$, that is,

$$
l(\alpha)=\frac{d \mu_{Y_{H}^{\alpha}}}{d \mu_{Y_{H}^{0}}}\left(Y_{H}^{\alpha}\right)=\exp \left[\alpha \int_{0}^{M} Q_{H}^{\alpha}(t) d Z_{H}^{\alpha}(t)-\frac{\alpha^{2}}{2} \int_{0}^{M}\left\{Q_{H}^{\alpha}(t)\right\}^{2} d v_{H}(t)\right] .
$$


Maximizing $l(\alpha)$ yields the MLE

$$
\tilde{\alpha}_{M L E}=\frac{\int_{0}^{M} Q_{H}^{\alpha}(t) d Z_{H}^{\alpha}(t)}{\int_{0}^{M}\left\{Q_{H}^{\alpha}(t)\right\}^{2} d v_{H}(t)}=\frac{U(H, M, \alpha)}{V(H, M, \alpha)},
$$

where

$$
U(H, M, \alpha)=\int_{0}^{M} Q_{H}^{\alpha}(t) d Z_{H}^{\alpha}(t), \quad V(H, M, \alpha)=\int_{0}^{M}\left\{Q_{H}^{\alpha}(t)\right\}^{2} d v_{H}(t) .
$$

Note that, when $\alpha=0$ and $H=1 / 2$, we have

$$
\begin{aligned}
\tilde{\alpha}_{M L E} & =\frac{\int_{0}^{M} Q_{1 / 2}^{0}(t) d Z_{1 / 2}^{0}(t)}{\int_{0}^{M}\left\{Q_{1 / 2}^{0}(t)\right\}^{2} d v_{1 / 2}(t)} \\
& =\frac{\int_{0}^{M} W(t) d W(t)}{\int_{0}^{M} W^{2}(t) d t} \stackrel{\mathcal{D}}{=} \frac{1}{M} \frac{\int_{0}^{1} W(t) d W(t)}{\int_{0}^{1} W^{2}(t) d t}, \quad(\alpha=0, H=1 / 2),
\end{aligned}
$$

which is also the OLSE under this situation.

We now consider

$$
F_{M L E}(x)=P\left(\tilde{\alpha}_{M L E}<x\right)=P(x V(M, H, \alpha)-U(M, H, \alpha)>0) .
$$

To compute $F_{M L E}(x)$ we need the joint moment generating function (m.g.f.) of $U(M, H, \alpha)$ and $V(M, H, \alpha)$, which we can derive from Kleptysna and Le Breton (2002) and the fractional version of Girsanov's theorem described above. We have

Thorem 1. The joint m.g.f. $m\left(\theta_{1}, \theta_{2}\right)$ of $U(M, H, \alpha)$ and $V(M, H, \alpha)$ is given by

$$
\begin{aligned}
m\left(\theta_{1}, \theta_{2}\right)=\mathrm{E}\left[\operatorname { e x p } \left\{\theta_{1} U\right.\right. & \left.\left.(H, M, \alpha)+\theta_{2} V(H, M, \alpha)\right\}\right] \\
=e^{-M\left(\alpha+\theta_{1}\right) / 2} & {\left[\left(1+\frac{\left(\alpha+\theta_{1}\right)^{2}}{\mu^{2}}\right) \cosh ^{2} \frac{\mu M}{2}-\frac{\alpha+\theta_{1}}{\mu} \sinh \mu M\right.} \\
& +\frac{\pi M}{4 \sin \pi H}\left\{-\frac{\left(\alpha+\theta_{1}\right)^{2}}{\mu} I_{-H}\left(\frac{\mu M}{2}\right) I_{H-1}\left(\frac{\mu M}{2}\right)\right. \\
& \left.\left.+\mu I_{1-H}\left(\frac{\mu M}{2}\right) I_{H}\left(\frac{\mu M}{2}\right)\right\}\right]^{-1 / 2},
\end{aligned}
$$

where $\mu=\sqrt{\alpha^{2}-2 \theta_{2}}$, whereas $I_{\nu}(z)$ is the modified Bessel function of the first kind defined by

$$
I_{\nu}(z)=\sum_{k=0}^{\infty} \frac{(z / 2)^{\nu+2 k}}{k ! \Gamma(\nu+k+1)} .
$$

Theorem 1 enables us to compute $F_{M L E}(x)$ employing Imhof's formula in the following way:

$$
F_{M L E}(x)=\frac{1}{2}+\frac{1}{\pi} \int_{0}^{\infty} \frac{1}{\theta} \operatorname{Im}[m(-i \theta, i \theta x)] d \theta,
$$

where $m(-i \theta, i \theta x)$ is the c.f. of $x V(H, M, \alpha)-U(M, H, \alpha)$. The computation of $F_{M L E}(x)$ will be done in the next section together with that of the probability density. The asymptotic distribution of $\tilde{\alpha}_{M L E}$ as $M \rightarrow \infty$ will also be derived. 
The MCE of $\alpha$ was derived in Bishwal (2008) as follows. The score function $s(\alpha)$ based on the likelihood $l(\alpha)$ in (30) is obtained as

$$
\begin{aligned}
s(\alpha) & =\frac{d \log l(\alpha)}{d \alpha}=\int_{0}^{M} Q_{H}^{\alpha}(t) d Z_{H}^{\alpha}(t)-\alpha \int_{0}^{M}\left\{Q_{H}^{\alpha}(t)\right\}^{2} d v_{H}(t) \\
& =\frac{1}{2}\left[\frac{\eta_{H}}{2(1-H)} Z_{H}^{\alpha}(M) \int_{0}^{M} t^{2 H-1} d Z_{H}^{\alpha}(t)-M\right]-\alpha \int_{0}^{M}\left\{Q_{H}^{\alpha}(t)\right\}^{2} d v_{H}(t),
\end{aligned}
$$

where this last equality is due to Kleptsyna and Le Breton (2002). Then the MCE is defined as the solution to the estimating equation

$$
e(\alpha)=-\frac{M}{2}-\alpha \int_{0}^{M}\left\{Q_{H}^{\alpha}(t)\right\}^{2} d v_{H}(t)=0,
$$

which yields the following MCE:

$$
\hat{\alpha}_{M C E}=\frac{-M / 2}{\int_{0}^{M}\left\{Q_{H}^{\alpha}(t)\right\}^{2} d v_{H}(t)} .
$$

It is seen that the support of $\hat{\alpha}_{M C E}$ is nonpositive, which may be desirable because $\alpha$ is also assumed to be nonpositive.

It follows from Theorem 1 that the distribution of $\hat{\alpha}_{M C E}$ is computed as

$$
\begin{aligned}
F_{M C E}(x) & =P\left(\hat{\alpha}_{M C E}<x\right)=P(x V(H, M, \alpha)+M / 2>0) \\
& =\frac{1}{2}+\frac{1}{\pi} \int_{0}^{\infty} \frac{1}{\theta} \operatorname{Im}\left[e^{i M \theta / 2} m(0, i \theta x)\right] d \theta,
\end{aligned}
$$

where $e^{i M \theta / 2} m(0, i \theta x)$ is the c.f. of $x V(H, M, \alpha)+M / 2$. The computation of $F_{M C E}(x)$ will be done in the next section together with the derivation of its asymptotic distribution as $M \rightarrow \infty$.

\section{Computation of the distributions of the MLE and MCE}

In this section we first compute the distribution functions of the MLE and MCE together with their moments. Then the asymptotic distributions of the two estimators as the sampling span $M$ becomes large will be derived. We also compare these estimators with the OLSE in terms of the asymptotic relative efficiency.

The distribution functions $F_{M L E}$ in (37) and $F_{M C E}$ in (39) of $\tilde{\alpha}_{M L E}$ and $\hat{\alpha}_{M C E}$, respectively, can be computed numerically using Simpson's rule. The probability densities of these distributions can also be computed by numerical differentiation of the distribution functions. Care, however, needs to be taken in the computation of the c.f.s because they contain the square roots of complex-valued quantities. To overcome this difficulty a modified algorithm as shown in Tanaka (1996) may be necessary.

In the actual computations, we used the change of variables formula. More specifically, in computing (37), we used

$$
\int_{0}^{\infty} \frac{1}{\theta} \operatorname{Im}[m(-i \theta, i \theta x)] d \theta=2 \int_{0}^{\infty} \frac{1}{u} \operatorname{Im}\left[m\left(-i u^{2}, i u^{2} x\right)\right] d u .
$$

This makes the numerical computation faster and the computation of the integrand at the origin unnecessary.

The c.f.s involve $I_{\nu}(z)$, the modified Bessel function of the first kind, the computaion of which may be troublesome if $\nu$ is negative or $z$ is complex because some software packages 
do not allow for this case. In that case we can proceed as follows. When $\nu$ is negative, but $\nu+1$ is positive, we use the following relation (Watson 1958):

$$
I_{\nu}(z)=\frac{2(\nu+1)}{z} I_{\nu+1}(z)+I_{\nu+2}(z) .
$$

When $z$ is complex, we can further use the following relation:

$$
I_{\nu}(z)=i^{-\nu} J_{\nu}(i z)
$$

where $J_{\nu}(z)$ is the Bessel function of the first kind defined by

$$
J_{\nu}(z)=\sum_{k=0}^{\infty} \frac{(-1)^{k}(z / 2)^{\nu+2 k}}{k ! \Gamma(\nu+k+1)}
$$

It is assumed here that, when $z$ is complex, the computation of $I_{\nu}(z)$ is not available, but that of $J_{\nu}(z)$ is available. Thus, to deal with the modified Bessel funcions of the first kind involved in the c.f.s, we can compute them as

$$
\begin{aligned}
I_{-H}(\xi) I_{H-1}(\xi)= & -\frac{4 H(1-H)}{\xi^{2}} i J_{1-H}(i \xi) J_{H}(i \xi)-\frac{2(1-H)}{\xi} J_{H+1}(i \xi) J_{1-H}(i \xi) \\
& -\frac{2 H}{\xi} J_{2-H}(i \xi) J_{H}(i \xi)+i J_{2-H}(i \xi) J_{H+1}(i \xi), \\
I_{1-H}(\xi) I_{H}(\xi)= & -i J_{1-H}(i \xi) J_{H}(i \xi),
\end{aligned}
$$

where $\xi=M \sqrt{\alpha^{2}-2 i \theta x} / 2$.

Figure 1 draws the probability densities of $\tilde{\alpha}_{M L E}$ for various values of $M$ when $\alpha=0$ and $H=1 / 2$, that is, densities given in (33). It is seen that the distribution tends to be concentrated around $\alpha=0$ as $M$ becomes large, which is also implied by the last expression in (33). Though not shown here, the distributions shrink to some extent as $H$ becomes large. Figure 2 is concerned with the probability densities of $\hat{\alpha}_{M C E}$ when $\alpha=0$ and $H=1 / 2$. The distributions are quite different from those of $\tilde{\alpha}_{M L E}$. They have a negative support only and tends to be monotonically increasing as $M$ becomes large. Though not shown here, the distributions are shifted to the right with smaller variances as $H$ becomes large.

\section{Figure $1 \quad$ Figure 2}

When $\alpha=0$ and $H$ is arbitrary, there is an exact relationship among the percent points of each of two estimators under various sampling spans $M$, which we describe as

Theorem 2. Assume that the true value of $\alpha$ is 0 , and let $\tilde{\alpha}_{M L E}(H, M)$ and $\hat{\alpha}_{M C E}(H, M)$ be the MLE and MCE under $H$ and $M$, respectively. Then it holds that

$$
\tilde{\alpha}_{M L E}(H, M) \stackrel{\mathcal{D}}{=} \tilde{\alpha}_{M L E}(H, 1) / M, \quad \hat{\alpha}_{M C E}(H, M) \stackrel{\mathcal{D}}{=} \hat{\alpha}_{M C E}(H, 1) / M .
$$

Theorem 2 implies that, when $\alpha=0$, there also exist the relations $x_{\gamma}(H, M)=x_{\gamma}(H, 1) / M$ and $y_{\gamma}(H, M)=y_{\gamma}(H, 1) / M$, where $x_{\gamma}(H, M)$ and $y_{\gamma}(H, M)$ are the $100 \gamma \%$ points of the distributions of $\tilde{\alpha}_{M L E}$ and $\hat{\alpha}_{M C E}$, respectively, under $H$ and $M$. 
Figures 3 and 4 are concerned with distributions of $\tilde{\alpha}_{M L E}$ for nonzero $\alpha$. The former is for $\alpha=-3, H=0.7$, whereas the latter for $\alpha=-5, H=0.9$. It is seen that the distributions tend to normality as $M$ becomes large, unlike the case $\alpha=0$, and the variation is larger for $\alpha$ smaller. The distributions do depend on $\alpha$, but little on $H$, although not shown here. This last fact will be theoretically confirmed shortly. The distributions of $\hat{\alpha}_{M C E}$ are found to be quite close to those of $\tilde{\alpha}_{M L E}$ in the present case as $M$ becomes large, which is also verified now.

\section{Figure 3}

\section{Figure 4}

The following theorem describes the asymptotic distributions of two estimators as $M \rightarrow \infty$.

Theorem 3. When $\alpha<0$, both $\tilde{\alpha}_{M L E}$ and $\hat{\alpha}_{M C E}$ are $\sqrt{M}$-consistent and it holds that, as $M \rightarrow \infty$,

$$
\sqrt{M}\left(\tilde{\alpha}_{M L E}-\alpha\right) \rightarrow \mathrm{N}(0,-2 \alpha), \quad \sqrt{M}\left(\tilde{\alpha}_{M C E}-\alpha\right) \rightarrow \mathrm{N}(0,-2 \alpha) .
$$

On the other hand, when $\alpha=0$, the asymptotic distributions of two estimators are different, but they are $M$-consistent, and $M \tilde{\alpha}_{M L E}$ and $M \hat{\alpha}_{M C E}$ have non-degenerate distributions.

Table 1 reports the means and variances of $\tilde{\alpha}_{M L E}$ and $\hat{\alpha}_{M C E}$. Note that we can use the following formulas to compute moments of these estimators:

$$
\begin{aligned}
& \mathrm{E}\left(\tilde{\alpha}_{M L E}^{k}\right)=\left.\frac{1}{(k-1) !} \int_{0}^{\infty} \theta_{2}^{k-1} \frac{\partial m\left(\theta_{1},-\theta_{2}\right)}{\partial \theta_{1}^{k}}\right|_{\theta_{1}=0} d \theta_{2}, \\
& \mathrm{E}\left(\tilde{\alpha}_{M C E}^{k}\right)=\left(\frac{-M}{2}\right)^{k} \frac{1}{(k-1) !} \int_{0}^{\infty} \theta_{2}^{k-1} m\left(0,-\theta_{2}\right) d \theta_{2},
\end{aligned}
$$

where $m\left(\theta_{1}, \theta_{2}\right)$ is the joint m.g.f. defined in (35). In the actual computation, we applied the change of variables formula to put $\sqrt{\alpha^{2}+2 \theta_{2}}=x-\alpha$, that is, $\theta_{2}=x^{2} / 2-\alpha x$ so that $d \theta_{2}=(x-\alpha) d x$

\section{Table 1}

Table 1 confirms what was mentioned before. These may be summarized as

i) When $\alpha=0$, it holds that the means of $\tilde{\alpha}_{M L E}$ and $\hat{\alpha}_{M C E}$ under $M$ are equal to those under $M=1$ divided by $M$, respectively. The corresponding variances reduce to those dvided by $M^{2}$. These facts come from Theorem 2 .

ii) When $\alpha<0$, the means and variances of the two estimators are quite close to each other, especially when $M$ is large. They tend to be independent of $H$. This is because it holds that both $\tilde{\alpha}_{M L E}$ and $\hat{\alpha}_{M C E}$ tend to $\mathrm{N}(\alpha,-2 \alpha / M)$, which does not depend on $H$. 
It is also of interest to compare the efficiency of the OLSE $\hat{\alpha}_{1}$ in (15) and the practical estimator $\hat{\alpha}_{3}$ in (22) with the MLE $\tilde{\alpha}_{M L E}$. For this purpose we obtain, from (19), (23) and (42), the asymptotic relative efficiencies for $1 / 2<H<3 / 4$ as follows:

$$
\operatorname{eff}_{1}=\lim _{M \rightarrow \infty} \frac{\mathrm{V}\left(\sqrt{M}\left(\tilde{\alpha}_{M L E}-\alpha\right)\right)}{\mathrm{V}\left(\sqrt{M}\left(\hat{\alpha}_{1}-\alpha\right)\right)}=\frac{2}{\sigma_{H}^{2}}, \mathrm{eff}_{3}=\lim _{M \rightarrow \infty} \frac{\mathrm{V}\left(\sqrt{M}\left(\tilde{\alpha}_{M L E}-\alpha\right)\right)}{\mathrm{V}\left(\sqrt{M}\left(\hat{\alpha}_{3}-\alpha\right)\right)}=\frac{8 H^{2}}{\sigma_{H}^{2}},
$$

where $\sigma_{H}^{2}$ is defined in (19).

Figure 5 draws eff 1 and eff $_{3}$ for $1 / 2<H<3 / 4$. It is seen that the relative efficiencies of $\hat{\alpha}_{1}$ and $\hat{\alpha}_{3}$ decrease monotonically from 1 to 0 as $H$ approaches $H=3 / 4$, although the former is slightly better than the latter.

\section{Figure 5}

\section{The unit root test under the fBm}

In this section we deal with the testing problem about $\alpha$. Suppose that we are given the observations from the fO-U process (1), and consider

$$
H_{0}: \alpha=0 \quad \text { vs. } \quad H_{1}: \alpha<0 .
$$

In terms of the discrete-time near unit root process (8), this problem may be interpreted as testing if the coefficient $\rho=1+(\alpha / T)$ is equal to unity. Thus the above testing problem may be referred to as the unit root test.

We conduct the unit root tests based on $\tilde{\alpha}_{M L E}$ and $\hat{\alpha}_{M C E}$. Table 2 reports percent points of the null distributions of the two estimators under various values of $H$ with $M=1$ fixed. The distributions of $\tilde{\alpha}_{M L E}$ shrink to some extent as $H$ becomes large so that, as $H$ becomes large, the percent points below the median increase, whereas those above the median decrease. The distributions of $\hat{\alpha}_{M C E}$ are continually shifted to the right as $H$ becomes large so that the percent points monotonically increase with $H$. Note that the percent points under $M>1$ can be restored from those under $M=1$ by dividing the corresponding value by $M$, as Theorem 2 ensures.

\section{Table 2}

As for the power properties of the unit root tests considered here, we have

Theorem 4. Given $H$, the powers of the unit root tests based on $\tilde{\alpha}_{M L E}$ and $\hat{\alpha}_{M C E}$ depend only on $\alpha \times M$.

Due to Theorem 4 we have only to examine powers of the unit root tests in terms of the values of $\alpha \times M$. Table 3 reports powers at the $5 \%$ significance level for various values of $\alpha \times M$ under $H=0.5,0.7,0.9$. It is seen that the test based on the MLE dominates, but the difference is only slight. It is also noted that the powers are higher when $H$ is larger. Figure 6 draws the power functions of the two tests when $H=0.9$. We note inpassing that, when $H=0.5$, the test based on the MLE gives completely the same limiting local power of the usual unit root test under $\rho=1+(\alpha / T)$ as $T \rightarrow \infty$ (Tanaka 1996, p. 347). 


\section{Table $3 \quad$ Figure 6}

\section{Concluding remarks}

We have discussed the MLE anc MCE estimation of the drift parameter $\alpha$ in the fO-U process. Distribution functions of theses estimators have been computed together with their moments. It turned out that the distributions are quite different between $\alpha=0$ and $\alpha<0$. We have also derived the asymptotic distributions as the sampling span $M$ becomes large. It was found that, when $\alpha<0$, the two estimators are $\sqrt{M}$-consistent and tend to normality, whereas, when $\alpha=0$, the estimators multiplied by $M$ become nondegenerate distributions independent of $M$ so that they are $M$-consistent.

We also considered the testing problem $H_{0}: \alpha=0$ against $H_{1}: \alpha<0$, which may be interpreted as the unit root test in the discrete-time near unit root process. We have conducted the tests based on the MLE and MCE, and computed the powers. It was found that the powers depend only on $\alpha \times M$.

The MLE and MCE have further been compared with the OLSE in terms of the asymptotic relative efficiency. It is desirable to study the exact distributional property of the OLSE, for which the derivation of the associated c.f. is necessary. This seems difficult and remains to be done. 
Abramowitz, M. \& I.A. Stegun (1965) Handbook of Mathematical Functions, New York: Dover.

Bishwal, J.P.N. (2008) Parameter Estimation in Stochastic Differential Equations, Berlin: Springer-Verlag.

Cheridito P., H. Kawaguchi \& M. Maejima (2003) Fractional Ornstein-Uhlenbeck processes. Electronic Journal of Probability 8, 1-14.

Duncan, T.E., Y. Hu \& B. Pasik-Duncan (2000) Stochastic calculus for fractional Brownian motion I. Theory. SIAM Journal of Control and Optimization 38, 582-612.

Gripenberg, G. \& I. Norros (1996) On the prediction of fractional Brownian motion. Journal of Applied Probability 33, 400-410.

Hu Y. \& D. Nualart (2010) Parameter estimation for fractional Ornstein-Uhlenbeck processes. Statistics and Probability Letters 80, 1030-1038.

Kleptsyna, M.L., A. Le Breton \& M.C. Roubaud (2000) Parameter estimation and optimal filtering for fractional type stochastic systems. Statistical Inference for Stochastic Processes 3, 173-182.

Kleptsyna, M.L. \& A. Le Breton (2002) Statistical analysis of the fractional OrnsteinUhlenbeck type process. Statistical Inference for Stochastic Processes 5, 229-248.

Kolmogorov, A.N. (1940) Wienersche Spiralen und einige andere interessante Kurven im Hilbertschen Raum. Dokl. Acad. Nauk. SSSR 26, 115-118.

Liptser, R.S. \& A.N. Shiryaev (1977) Statistics of Random Processes I: General Theory, New York: Springer-Verlag.

Mandelbrot, B.B. \& J.W. Van Ness (1968) Fractional Brownian motions, fractional noises and applications. SIAM Review 10, 422-437.

Norros, I., E. Valkeila \& J. Virtamo (1999) An elementary approach to a Girsanov formula and other analytical results on fractional Brownian motions. Bernoulli 5, 571-587.

Tanaka, K. (1996) Time Series Analysis: Nonstationary and Noninvertible Distribution Theory, New York: Wiley.

Watson, G.N. (1958) A Treatise on the Theory of Bessel Functions, Second Edition, London: Cambridge University Press. 


\section{Appendix}

Proof of Theorem 1. It follows from the fractional version of Girsanov's theorem that

$$
\begin{aligned}
m\left(\theta_{1}, \theta_{2}\right)= & \mathrm{E}\left[\exp \left\{\theta_{1} \int_{0}^{M} Q_{H}^{\alpha}(t) d Z_{H}^{\alpha}(t)+\theta_{2} \int_{0}^{M}\left\{Q_{H}^{\alpha}(t)\right\}^{2} d v_{H}(t)\right\}\right] \\
= & \mathrm{E}\left[\exp \left\{\theta_{1} \int_{0}^{M} Q_{H}^{\beta}(t) d Z_{H}^{\beta}(t)+\theta_{2} \int_{0}^{M}\left\{Q_{H}^{\beta}(t)\right\}^{2} d v_{H}(t)\right\}\right. \\
& \left.\quad \times \frac{d \mu_{Y_{H}^{\alpha}}}{d \mu_{Y_{H}^{\beta}}}\left(Y_{H}^{\beta}\right)\right] \\
= & \mathrm{E}\left[\operatorname { e x p } \left\{\left(\theta_{1}+\alpha-\beta\right) \int_{0}^{M} Q_{H}^{\beta}(t) d Z_{H}^{\beta}(t)\right.\right. \\
& \left.\left.\quad+\left(\theta_{2}-\frac{\alpha^{2}-\beta^{2}}{2}\right) \int_{0}^{M}\left\{Q_{H}^{\beta}(t)\right\}^{2} d v_{H}(t)\right\}\right] \\
= & \mathrm{E}\left[\exp \left\{\left(\theta_{1}+\alpha-\beta\right) \int_{0}^{M} Q_{H}^{\beta}(t) d Z_{H}^{\beta}(t)\right\}\right] \\
= & e^{M\left(\beta-\alpha-\theta_{1}\right) / 2} \mathrm{E}\left[\exp \left\{\kappa Z_{H}^{\beta}(M) \int_{0}^{M} t^{2 H-1} d Z_{H}^{\beta}(t)\right\}\right]
\end{aligned}
$$

where $\kappa=\left(\theta_{1}+\alpha-\beta\right) \eta_{H} /(4(1-H))$ and we have put $\beta=\sqrt{\alpha^{2}-2 \theta_{2}}$. Then it follows from Kleptsyna and Le Breton (2002) that this last quantity yields

$$
\begin{aligned}
m\left(\theta_{1}, \theta_{2}\right)= & e^{M\left(\beta-\alpha-\theta_{1}\right) / 2}\left(\frac{4 \sin \pi H}{\pi \beta M}\right)^{1 / 2}\left[\left(1+\frac{\beta-\alpha-\theta_{1}}{\beta} e^{z} \sinh z\right)^{2} I_{-H}(z) I_{H-1}(z)\right. \\
& \left.-\left(1-\frac{\beta-\alpha-\theta_{1}}{\beta} e^{z} \cosh z\right)^{2} I_{1-H}(z) I_{H}(z)\right]^{-1 / 2} \\
= & e^{M\left(-\alpha-\theta_{1}\right) / 2}\left(\frac{4 \sin \pi H}{\pi M}\right)^{1 / 2}\left[\frac{1}{\beta}\left(\beta \cosh z-\left(\alpha+\theta_{1}\right) \sinh z\right)^{2} I_{-H}(z) I_{H-1}(z)\right. \\
& \left.-\frac{1}{\beta}\left(\beta \sinh z-\left(\alpha+\theta_{1}\right) \cosh z\right)^{2} I_{1-H}(z) I_{H}(z)\right]^{-1 / 2},
\end{aligned}
$$

where $z=\beta M / 2$. Then, using the relation

$$
I_{H}(z) I_{H-1}(z)-I_{1-H}(z) I_{H}(z)=\frac{4 \sin \pi H}{2 \pi z},
$$

it can be verified that $m\left(\theta_{1}, \theta_{2}\right)$ is given by (35), which establishes Theorem 1 .

Proof of Theorem 2. When $\alpha=0$, the distibution function of $\tilde{\alpha}_{M L E}$ is defined by

$$
\begin{aligned}
F_{M L E}(x) & =\frac{1}{2}+\frac{1}{\pi} \int_{0}^{\infty} \frac{1}{\theta} \operatorname{Im}[m(-i \theta, i \theta x)] d \theta \\
& =\frac{1}{2}+\frac{1}{\pi} \int_{0}^{\infty} \frac{1}{u} \operatorname{Im}[m(-i u / M, i u x / M)] d u
\end{aligned}
$$


where

$$
\begin{aligned}
m(-i u / M, i u x / M)= & e^{i u / 2}\left[\left(1-\frac{i u}{2 M x}\right) \cosh ^{2} z+u \frac{\sinh 2 z}{2 z}\right. \\
& \left.+\frac{\pi}{4 \sin \pi H}\left(2 z I_{1-H}(z) I_{H}(z)+\frac{u^{2}}{2 z} I_{-H}(z) I_{H-1}(z)\right)\right]^{-1 / 2}, \\
z= & \frac{1}{2} \sqrt{-2 i u M x} .
\end{aligned}
$$

Then, it follows from the form of the integrand, where $x$ is always coupled with $M$ as $M \times x$, we can confirm the statement in the theorem.

As for the MCE, we have

$$
\begin{aligned}
F_{M C E}(x) & =\frac{1}{2}+\frac{1}{\pi} \int_{0}^{\infty} \frac{1}{\theta} \operatorname{Im}\left[e^{i M \theta / 2} m(0, i \theta x)\right] d \theta \\
& =\frac{1}{2}+\frac{1}{\pi} \int_{0}^{\infty} \frac{1}{u} \operatorname{Im}\left[e^{i u / 2} m(0, i u x / M)\right] d u
\end{aligned}
$$

where

$$
m(0, i u x / M)=e^{i u / 2}\left[\cosh ^{2} z+\frac{\pi}{4 \sin \pi H} 2 z I_{1-H}(z) I_{H}(z)\right]^{-1 / 2} .
$$

The same reasoning as before applies here, which establishes Theorem 2.

Proof of Theorem 3. The MLE $\tilde{\alpha}_{M L E}$ is defined in (31) as $U(H, M, \alpha) / V(H, M, \alpha)=$ $U / V$. Suppose first that $\alpha<0$ and let us consider the joint m.g.f. $n_{1}\left(\theta_{1}, \theta_{2}\right)$ of $(U-$ $\alpha V) / \sqrt{M}$ and $V / M$, which is, from Theorem 1 ,

$$
\begin{aligned}
n_{1}\left(\theta_{1}, \theta_{2}\right)= & m\left(\frac{\theta_{1}}{\sqrt{M}}, \frac{\theta_{2}}{M}-\frac{\alpha \theta_{1}}{\sqrt{M}}\right) \\
= & {\left[e ^ { \sqrt { M } \theta _ { 1 } + \alpha M } \left\{\left(1+\frac{\left(\theta_{1} / \sqrt{M}+\alpha\right)^{2}}{\mu^{2}}\right) \cosh ^{2} z-\frac{\theta_{1} / \sqrt{M}+\alpha}{\mu} \sinh 2 z\right.\right.} \\
& \left.\left.+\frac{\pi M}{4 \sin \pi H}\left(\mu I_{1-H}(z) I_{H}(z)-\frac{\left(\theta_{1} / \sqrt{M}+\alpha\right)^{2}}{\mu} I_{-H}(z) I_{H-1}(z)\right)\right\}\right]^{-1 / 2},
\end{aligned}
$$

where

$$
\begin{aligned}
\mu & =\left(\alpha^{2}-2 \theta_{2} / M+2 \alpha \theta_{1} / \sqrt{M}\right)^{1 / 2}=-\alpha-\frac{\theta_{1}}{\sqrt{M}}+\frac{\theta_{2}}{\alpha M}+\frac{\theta_{1}^{2}}{2 \alpha M}+o\left(M^{-1}\right), \\
z & =\frac{1}{2} \mu M=\frac{1}{2}\left(-\alpha M-\sqrt{M} \theta_{1}+\frac{\theta_{2}}{\alpha}+\frac{\theta_{1}^{2}}{2 \alpha}\right)+o(1) .
\end{aligned}
$$

Since it holds (Abramowitz and Stegun 1965) that, as $M \rightarrow \infty$,

$$
I_{\nu}(z)=\frac{e^{z}}{\sqrt{2 \pi z}}\left(1+O\left(z^{-1}\right)=\frac{e^{\mu M / 2}}{\sqrt{\pi \mu M}}\left(1+O\left(M^{-1}\right)\right),\right.
$$

for fixed $\nu$ and, evidently,

$$
\cosh ^{2}(z)=\frac{1}{4} e^{\mu M}+O(1), \quad \sinh 2 z=\frac{1}{2} e^{\mu M}+O(1),
$$


we have

$$
\begin{aligned}
\mu & =\left[e^{\theta_{2} / \alpha+\theta_{1}^{2} /(2 \alpha)}\left\{\frac{1}{4}\left(1+\frac{\alpha^{2}+o(1)}{\alpha^{2}+o(1)}\right)-\frac{1}{2} \frac{\alpha+o(1)}{-\alpha+o(1)}+\frac{1}{4 \sin \pi H}\left(1-\frac{\alpha^{2}+o(1)}{\alpha^{2}+o(1)}\right)\right\}\right]^{-1 / 2} \\
& \rightarrow \exp \left\{\frac{\theta_{2}}{(-2 \alpha)}+\frac{1}{2} \frac{\theta_{1}^{2}}{(-2 \alpha)}\right\} .
\end{aligned}
$$

Thus we conclude that

$$
\frac{U-\alpha V}{\sqrt{M}} \rightarrow \mathrm{N}(0,1 /(-2 \alpha)), \quad \frac{V}{M} \rightarrow 1 /(-2 \alpha),
$$

so that

$$
\sqrt{M}(\tilde{\alpha}-\alpha)=\frac{(U-\alpha V) / \sqrt{M}}{V / M} \rightarrow \mathrm{N}(0,-2 \alpha) .
$$

We consider next the case for $\alpha=0$. The joint m.g.f. $j_{1}\left(\theta_{1}, \theta_{2}\right)$ of $U / M$ and $V / M^{2}$ is given by

$$
\begin{aligned}
j_{1}\left(\theta_{1}, \theta_{2}\right)= & m\left(\theta_{1} / M, \theta_{2} / M^{2}\right) \\
= & e^{-\theta_{1} / 2}\left[\left(1-\frac{\theta_{1}^{2}}{2 \theta_{2}}\right) \cosh ^{2} w-\theta_{1} \frac{\sinh 2 w}{2 w}+\frac{\pi}{4 \sin \pi H}\right. \\
& \left.\times\left\{2 w I_{1-H}(w) I_{H}(w)-\frac{\theta_{1}^{2}}{2 w} I_{-H}(w) I_{H-1}(w)\right\}\right]^{-1 / 2},
\end{aligned}
$$

where $w=\sqrt{-2 \theta_{2}} / 2$. Then we can coclude that $M \times U / V=M \times \tilde{\alpha}_{M L E}$ is nondegenerate and is independent of $\mathrm{M}$, which implies that $\tilde{\alpha}_{M L E}$ is $M$-consistent.

The case of $\hat{\alpha}_{M C E}$ proceeds similarly. The definition of $\hat{\alpha}_{M C E}$ is given in (38) as

$$
\hat{\alpha}_{M C E}=\frac{-M / 2}{\int_{0}^{M}\left\{Q_{H}^{\alpha}(t)\right\}^{2} d v_{H}(t)} .
$$

Suppose that $\alpha<0$ and consider the joint m.g.f. $n_{2}\left(\theta_{1}, \theta_{2}\right)$ of $(-M / 2-\alpha V) / \sqrt{M}$ and $V / M$, which is given by

$$
\begin{aligned}
n_{2}\left(\theta_{1}, \theta_{2}\right)= & e^{-\theta_{1} \sqrt{M} / 2} m\left(0, \theta_{2} / M-\alpha \theta_{1} / \sqrt{M}\right) \\
= & {\left[e ^ { \sqrt { M } \theta _ { 1 } + M \alpha } \left\{\left(1+\frac{\alpha^{2}}{\mu^{2}}\right) \cosh ^{2} z-\frac{\alpha}{\mu} \sinh 2 z\right.\right.} \\
& \left.\left.\quad+\frac{\pi M}{4 \sin \pi H}\left(\mu I_{1-H}(z) I_{H}(z)-\frac{\alpha^{2}}{\mu} I_{-H}(z) I_{H-1}(z)\right)\right\}\right]^{-1 / 2} \\
= & {\left[\exp \left\{\frac{\theta_{2}}{\alpha}+\frac{\theta_{1}^{2}}{2 \alpha}\right\}\left\{\frac{1}{4}\left(1+\frac{\alpha^{2}}{\mu^{2}}\right)-\frac{\alpha}{2 \mu}+\frac{1}{4 \sin \pi H}\left(1-\frac{\alpha^{2}}{\mu^{2}}\right)\right\}+o(1)\right]^{-1 / 2} } \\
\rightarrow & \exp \left\{\frac{\theta_{2}}{(-2 \alpha)}+\frac{1}{2} \frac{\theta_{1}^{2}}{(-2 \alpha)}\right\}
\end{aligned}
$$

which ensures that $\sqrt{M}\left(\hat{\alpha}_{M C E}-\alpha\right)$ tends to $\mathrm{N}(0,-2 \alpha)$. 
When $\alpha=0$, the joint m.g.f. $j_{2}\left(\theta_{1}, \theta_{2}\right)$ of $(-M / 2) / M=-1 / 2$ and $V / M^{2}$ is given by

$$
\begin{aligned}
j_{2}\left(\theta_{1}, \theta_{2}\right) & =e^{-\theta_{1} / 2} m\left(0, \theta_{2} / M^{2}\right) \\
& =e^{-\theta_{1} / 2}\left[\cosh ^{2} w+\frac{\pi}{4 \sin \pi H} 2 w I_{1-H}(w) I_{H}(w)\right]^{-1 / 2},
\end{aligned}
$$

which implies that $M \hat{\alpha}_{M C E}$ is independent of $M$ and $\hat{\alpha}_{M C E}$ is $M$-consistent. Theorem 3 has now been established.

Proof of Theorem 4. Let $x_{\gamma}(H, M)$ be the $100 \gamma \%$ point of the distribution of $\tilde{\alpha}_{M L E}$ for $\alpha=0$ under $H$ and $M$. Then the power of the test at the level $\gamma$ is computed as

$$
\begin{aligned}
P\left(\tilde{\alpha}_{M L E}<x_{\gamma}(H, M) \mid \alpha<0\right) & =\frac{1}{2}+\frac{1}{\pi} \int_{0}^{\infty} \frac{1}{\theta} \operatorname{Im}\left[\phi\left(\theta ; x_{\gamma}(H, M)\right)\right] d \theta, \\
& =\frac{1}{2}+\frac{1}{\pi} \int_{0}^{\infty} \frac{1}{u} \operatorname{Im}\left[\phi\left(u / M ; x_{\gamma}(H, M)\right)\right] d u,
\end{aligned}
$$

where

$$
\begin{aligned}
\phi\left(u / M ; x_{\gamma}(H, M)\right)= & m\left(-i u / M, i u x_{\gamma}(H, m) / M\right) \\
= & e^{i u-\alpha M}\left[\left(1+\frac{(i u-\alpha M)^{2}}{4 z^{2}}\right) \cosh ^{2} z+(i u-\alpha M) \frac{\sinh 2 z}{2 z}\right. \\
& \left.\quad+\frac{\pi}{4 \sin \pi H}\left\{2 z I_{1-H}(z) I_{H}(z)-\frac{(i u-\alpha M)^{2}}{2 z} I_{-H}(z) I_{H-1}(z)\right\}\right]^{-1 / 2}, \\
z= & \frac{1}{2}\left((\alpha M)^{2}-2 i u M x_{\gamma}(H, M)\right)^{1 / 2} .
\end{aligned}
$$

Noting that $M x_{\gamma}(H, M)$ is independent of $M$ because of Theorem 2, it is seen from the form of the c.f. that the power depends only on $\alpha M$. We can also prove this fact for the power based on $\hat{\alpha}_{M C E}$, which establishes Theorem 4 . 
Table 1. Means and variances of $\tilde{\alpha}_{M L E}$ and $\hat{\alpha}_{M C E}$

\begin{tabular}{|c|c|c|c|c|c|c|}
\hline$(M, H)$ & $(1,0.5)$ & $(40,0.5)$ & $(1,0.7)$ & $(40,0.7)$ & $(1,0.9)$ & $(40,0.9)$ \\
\hline \multicolumn{7}{|c|}{$\alpha=0$} \\
\hline $\mathrm{E}\left(\tilde{\alpha}_{M L E}\right)$ & -1.781 & -0.045 & -1.763 & -0.044 & -1.578 & -0.039 \\
\hline $\mathrm{E}\left(\hat{\alpha}_{M C E}\right)$ & -2.781 & -0.070 & -2.671 & -0.067 & -2.094 & -0.052 \\
\hline $\mathrm{V}\left(\tilde{\alpha}_{M L E}\right)$ & 10.112 & 0.0063 & 9.924 & 0.0062 & 8.731 & 0.0055 \\
\hline $\mathrm{V}\left(\hat{\alpha}_{M C E}\right)$ & 9.221 & 0.0058 & 8.986 & 0.0056 & 7.601 & 0.0048 \\
\hline \multicolumn{7}{|c|}{$\alpha=-1$} \\
\hline $\mathrm{E}\left(\tilde{\alpha}_{M L E}\right)$ & -2.882 & -1.050 & -2.874 & -1.050 & -2.752 & -1.050 \\
\hline $\mathrm{E}\left(\hat{\alpha}_{M C E}\right)$ & -3.700 & -1.063 & -3.583 & -1.060 & -2.931 & -1.037 \\
\hline $\mathrm{V}\left(\tilde{\alpha}_{M L E}\right)$ & 11.761 & 0.056 & 11.551 & 0.056 & 10.284 & 0.054 \\
\hline $\mathrm{V}\left(\hat{\alpha}_{M C E}\right)$ & 11.329 & 0.056 & 11.106 & 0.056 & 9.771 & 0.056 \\
\hline \multicolumn{7}{|c|}{$\alpha=-3$} \\
\hline $\mathrm{E}\left(\tilde{\alpha}_{M L E}\right)$ & -4.954 & -3.050 & -4.953 & -3.050 & -4.895 & -3.050 \\
\hline $\mathrm{E}\left(\hat{\alpha}_{M C E}\right)$ & -5.626 & -3.063 & -5.506 & -3.060 & -4.769 & -3.035 \\
\hline $\mathrm{V}\left(\tilde{\alpha}_{M L E}\right)$ & 15.410 & 0.156 & 15.175 & 0.155 & 13.729 & 0.154 \\
\hline $\mathrm{V}\left(\hat{\alpha}_{M C E}\right)$ & 15.426 & 0.156 & 15.216 & 0.156 & 14.026 & 0.156 \\
\hline \multicolumn{7}{|c|}{$\alpha=-5$} \\
\hline $\mathrm{E}\left(\tilde{\alpha}_{M L E}\right)$ & -6.976 & -5.050 & -6.976 & -5.050 & -6.940 & -5.050 \\
\hline $\mathrm{E}\left(\hat{\alpha}_{M C E}\right)$ & -7.593 & -5.063 & -7.472 & -5.060 & -6.690 & -5.035 \\
\hline $\mathrm{V}\left(\tilde{\alpha}_{M L E}\right)$ & 19.272 & 0.256 & 19.031 & 0.255 & 17.480 & 0.254 \\
\hline $\mathrm{V}\left(\hat{\alpha}_{M C E}\right)$ & 19.460 & 0.256 & 19.253 & 0.256 & 18.183 & 0.256 \\
\hline
\end{tabular}


Table 2. Percent points of the distributions of $\tilde{\alpha}_{M L E}$ and $\hat{\alpha}_{M C E}$ for $\alpha=0$ and $M=1$

\begin{tabular}{|c|c|c|c|c|c|c|c|}
\hline \multirow{2}{*}{$\mathrm{H}$} & \multirow[b]{2}{*}{0.01} & \multicolumn{2}{|c|}{ Probability } & \multirow{2}{*}{$\begin{array}{r}\text { of } \quad \mathrm{a} \\
\\
0.5 \\
\end{array}$} & \multirow{2}{*}{$\begin{array}{r}\text { smaller } \\
0.9 \\
\end{array}$} & \multirow{2}{*}{$\begin{array}{l}\text { value } \\
0.95 \\
\end{array}$} & \multirow[b]{2}{*}{0.99} \\
\hline & & 0.05 & 0.1 & & & & \\
\hline & & & $\tilde{\alpha}_{M L E}$ & & & & \\
\hline 0.5 & -13.696 & -8.039 & -5.714 & -0.853 & 0.928 & 1.285 & 2.033 \\
\hline 0.6 & -13.676 & -8.023 & -5.699 & -0.850 & 0.921 & 1.277 & 2.022 \\
\hline 0.7 & -13.608 & -7.964 & -5.648 & -0.836 & 0.899 & 1.250 & 1.989 \\
\hline 0.8 & -13.446 & -7.822 & -5.523 & -0.789 & 0.856 & 1.195 & 1.924 \\
\hline 0.9 & -12.988 & -7.415 & -5.154 & -0.636 & 0.767 & 1.084 & 1.791 \\
\hline
\end{tabular}

\begin{tabular}{llllllll}
0.5 & -14.510 & -8.856 & -6.533 & -1.721 & -0.418 & -0.302 & -0.179 \\
0.6 & -14.465 & -8.814 & -6.493 & -1.695 & -0.409 & -0.295 & -0.175 \\
0.7 & -14.314 & -8.673 & -6.359 & -1.606 & -0.378 & -0.272 & -0.161 \\
0.8 & -13.983 & -8.364 & -6.067 & -1.420 & -0.317 & -0.228 & -0.134 \\
0.9 & -13.196 & -7.630 & -5.376 & -1.027 & -0.209 & -0.149 & -0.087 \\
\hline
\end{tabular}


Table 3. Percent powers of the unit root tests at the $5 \%$ level based on $\tilde{\alpha}_{M L E}$ and $\hat{\alpha}_{M C E}$

\begin{tabular}{lrrrrrr}
\hline$\alpha \times M$ & -0.5 & -1 & -5 & -10 & -15 & -20 \\
\hline$H=0.5$ & & & & & & \\
& & & & & & \\
$M L E$ & 6.30 & 7.86 & 31.42 & 75.57 & 96.94 & 99.88 \\
$M C E$ & 6.25 & 7.74 & 30.02 & 73.12 & 96.15 & 99.83 \\
& & & & & & \\
$H=0.7$ & & & & & & \\
$M L E$ & 6.32 & 7.89 & 31.86 & 76.37 & 97.20 & 99.90 \\
$M C E$ & 6.26 & 7.77 & 30.36 & 73.78 & 96.40 & 99.85 \\
& & & & & & \\
$H=0.9$ & & & & & & \\
$M L E$ & 6.41 & 8.13 & 35.26 & 82.20 & 98.62 & 99.97 \\
$M C E$ & 6.31 & 7.88 & 31.70 & 76.00 & 97.05 & 99.89 \\
& & & & & & \\
\hline
\end{tabular}




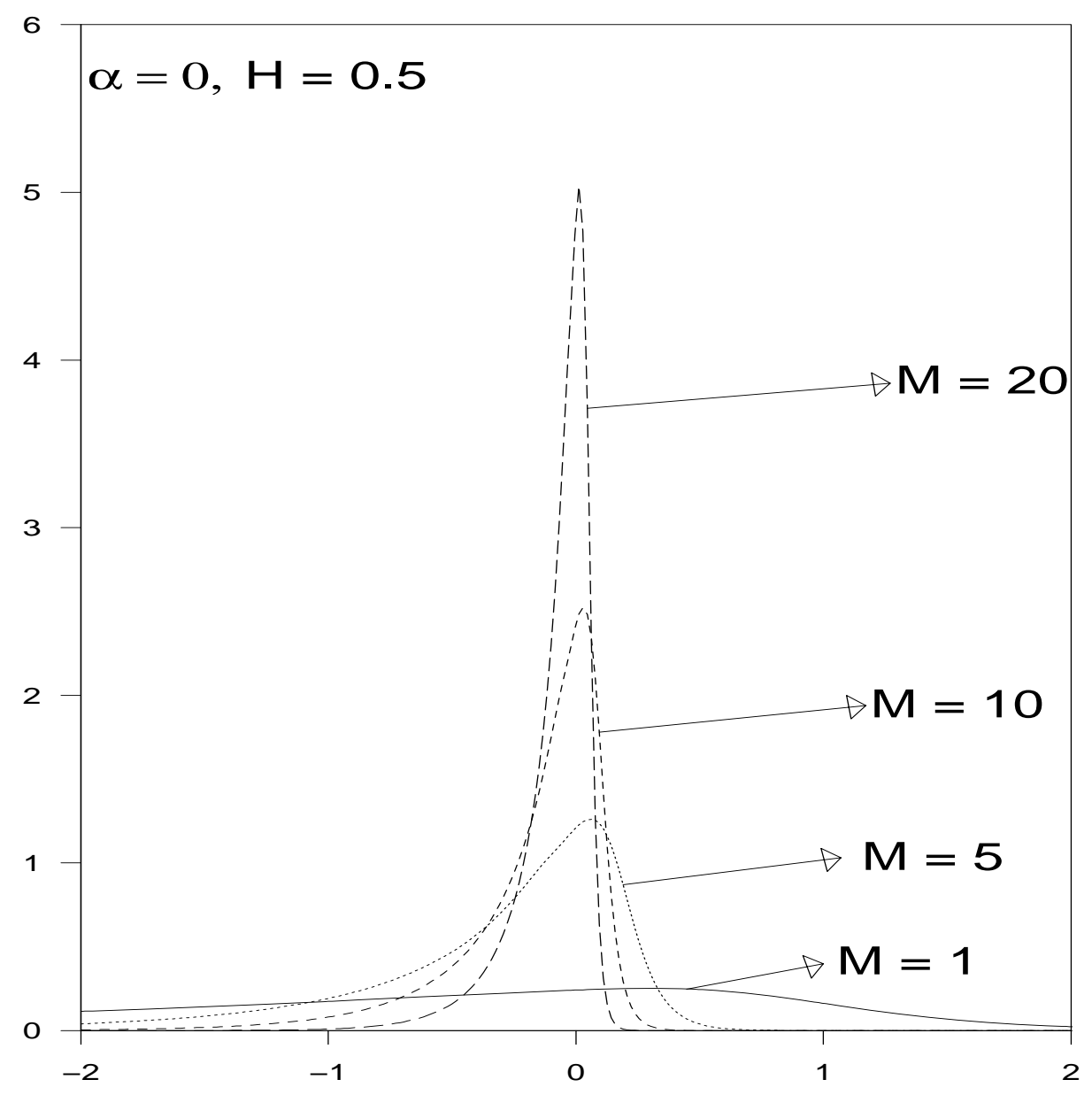

Figure 1. Probability densities of $\tilde{\alpha}_{M L E}$ when $\alpha=0$ and $H=1 / 2$ 


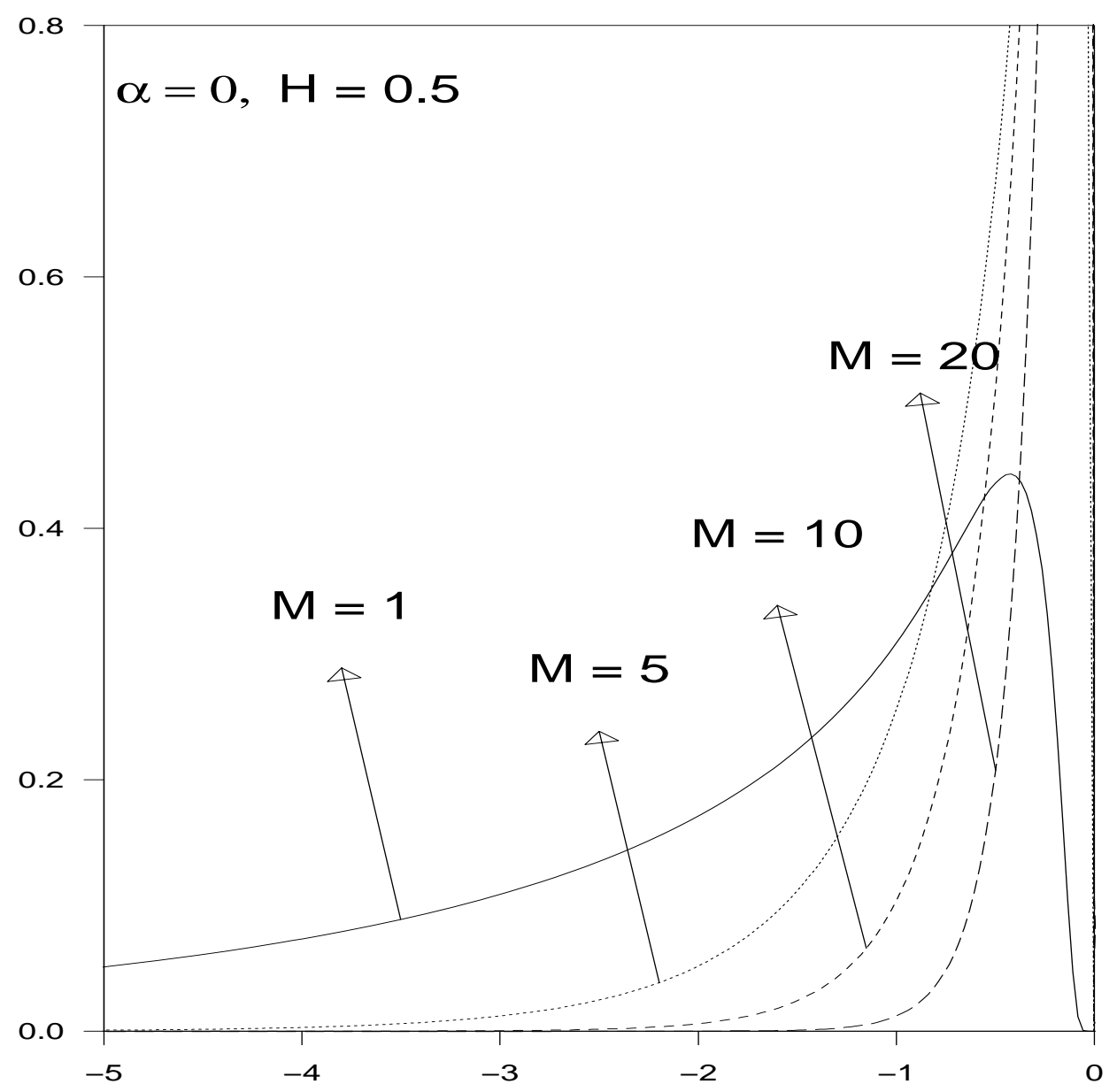

Figure 2. Probability densities of $\hat{\alpha}_{M C E}$ when $\alpha=0$ and $H=1 / 2$ 


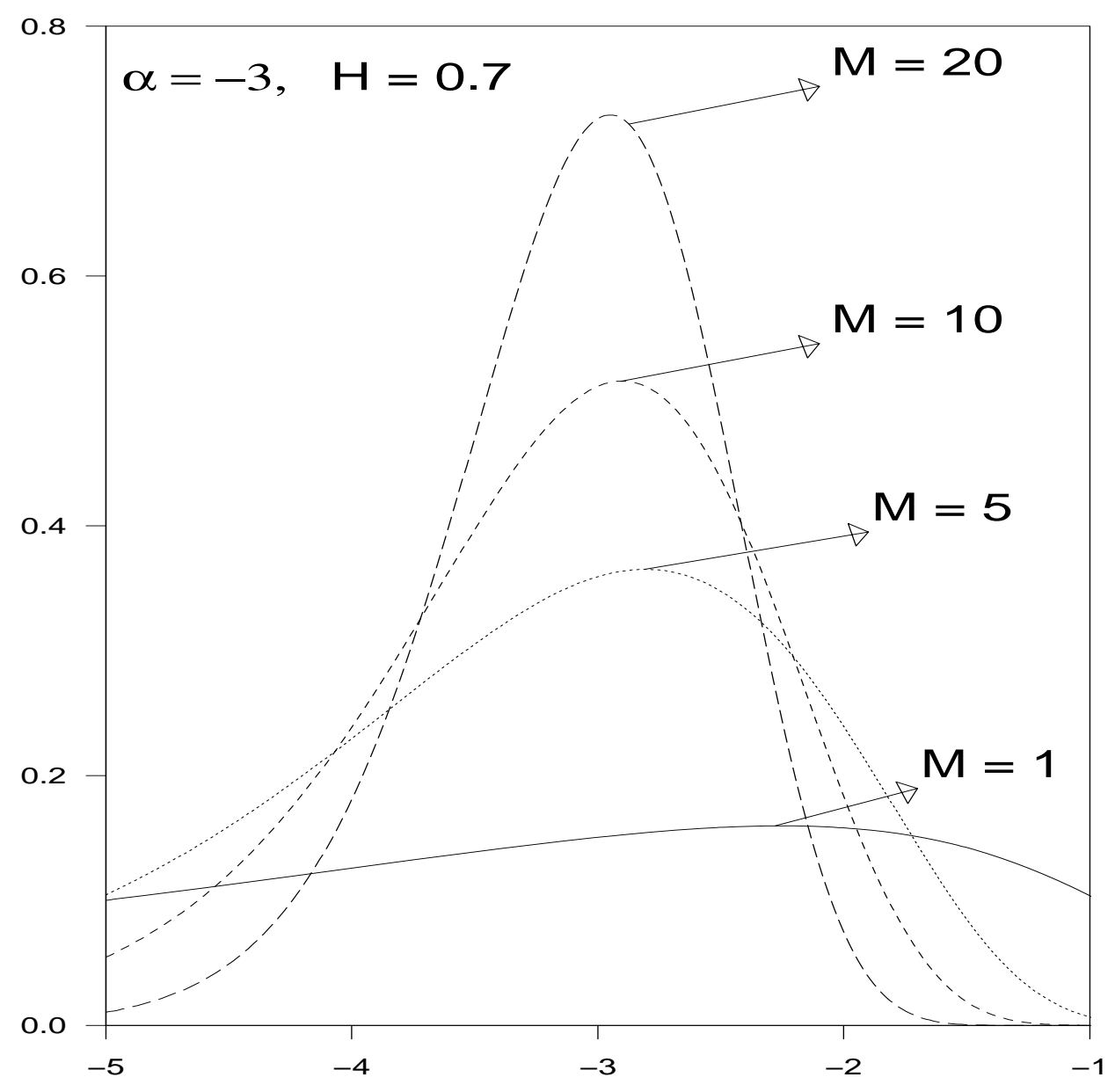

Figure 3. Probability densities of $\tilde{\alpha}_{M L E}$ when $\alpha=-3$ and $H=0.7$ 


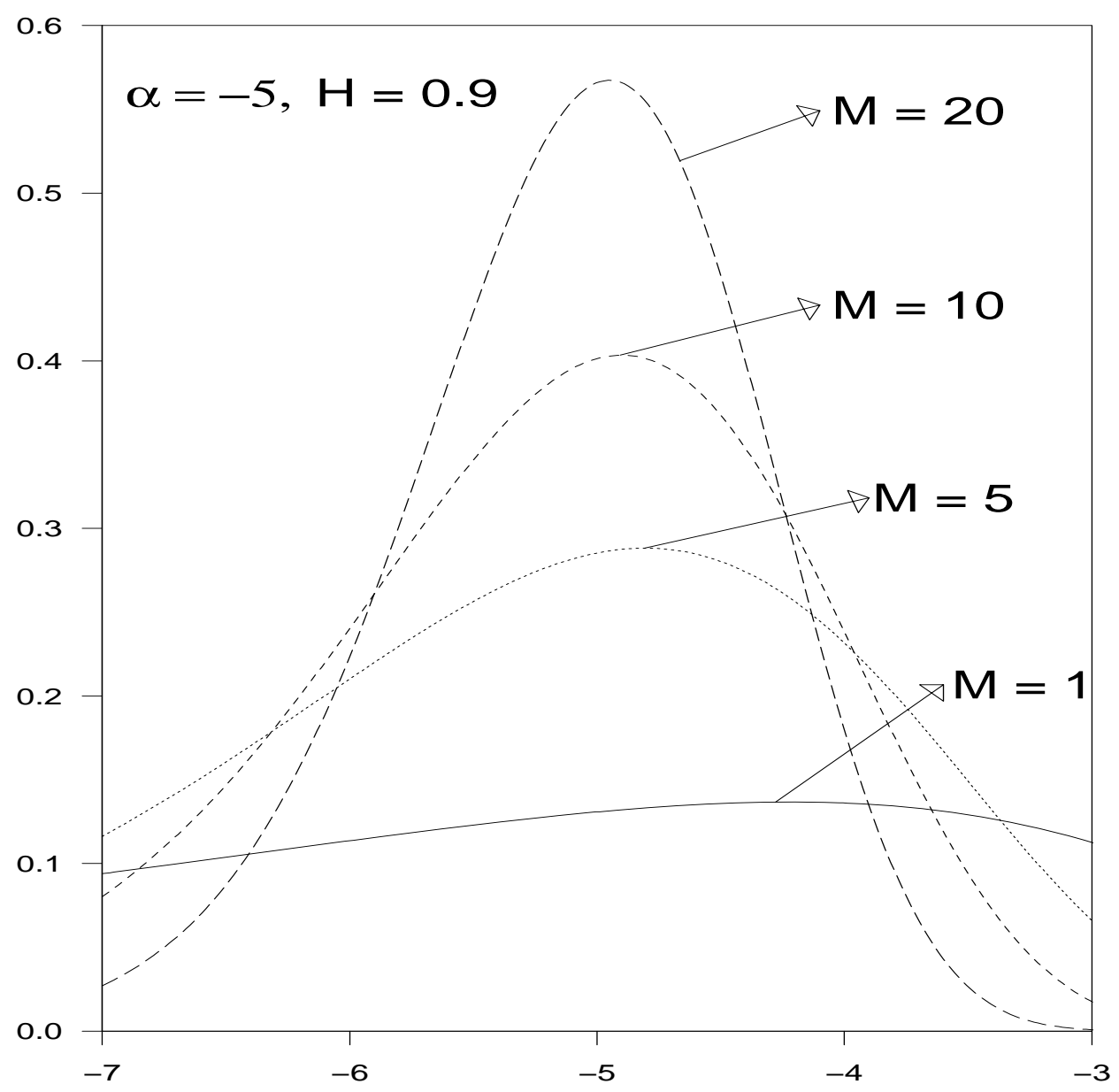

Figure 4. Probability densities of $\tilde{\alpha}_{M L E}$ when $\alpha=-5$ and $H=0.9$ 


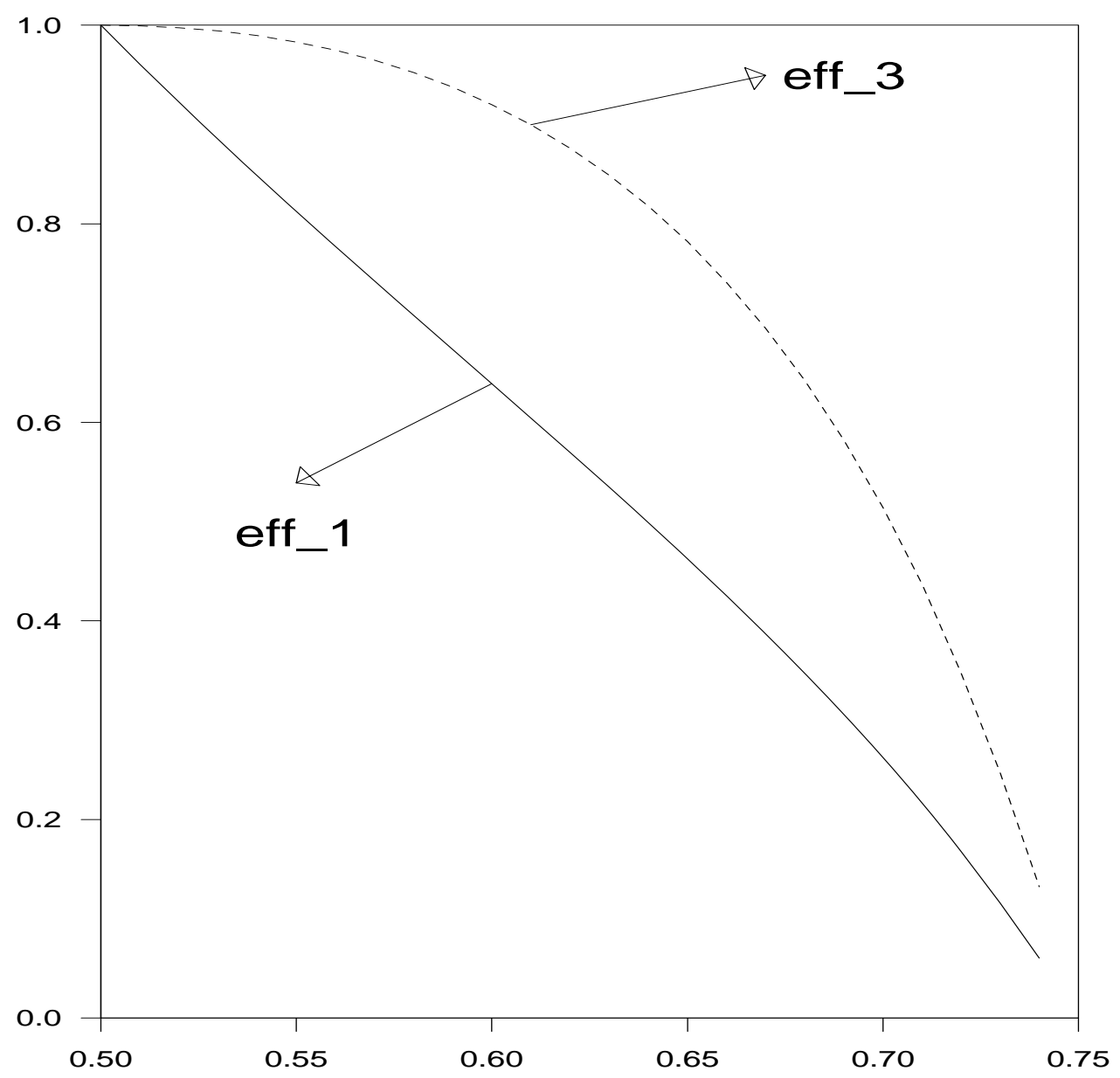

Figure 5. Asymptotic relative efficiencies of $\hat{\alpha}_{1}$ and $\hat{\alpha}_{3}$ 


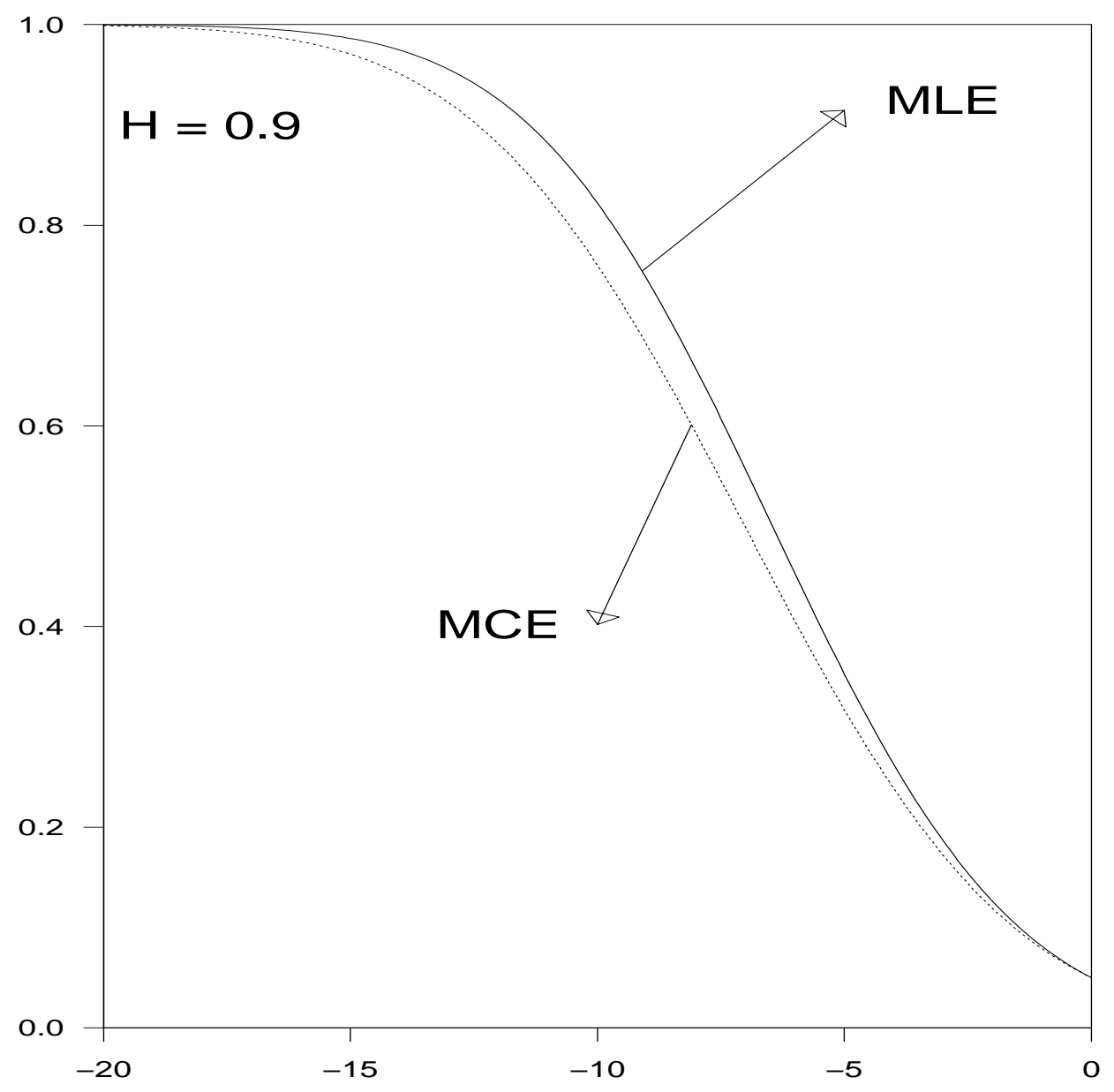

Figure 6 . Powers of the unit root tests at the $5 \%$ level based on $\tilde{\alpha}_{M L E}$ and $\hat{\alpha}_{M C E}$ 